\title{
THE GASEOUS CONTENT OF GALAXIES
}

\author{
MORTON S. ROBERTS \\ National Radio Astronomy Observatory,* Green Bank, West Va., U.S.A.
}

\begin{abstract}
A general review is given of the content and distribution of interstellar gas within galaxies. The constancy of the ratio $N(\mathrm{He}) / N(\mathrm{H})$, independent of galaxy type (spirals and irregulars), is discussed and the possible mechanisms for this constancy are considered. The helium abundance does not vary across the disk of spirals, although nitrogen and possibly other elements do.

The gross features of the neutral hydrogen distribution in our Galaxy and other systems are described. In spirals, the peak of the radial distribution of $\mathrm{HI}_{\mathrm{I}}$ is located well away from the optical center. This is not the case for irregular-type systems. A possible correlation of the relative location of the maxima of $\mathrm{HI}$ and $\mathrm{HII}$ distributions with galaxy type is described. Many spirals studied with high enough relative angular resolution show concentrations of $\mathrm{HI}_{\mathrm{I}}$ in their outermost regions. These may be due to hydrogen companions or warps in the hydrogen plane. Hydrogen 'bridges' are described and a new example for the triple system M81-M82-NGC 3077 is given. This latter case may be an extreme example of distortion by companion galaxies of the $\mathrm{HI}$ associated with a massive galaxy.

The neutral hydrogen content of a galaxy and its correlation with other integral properties is discussed. The absorption profile due to hydrogen associated with the radio galaxy Centaurus $A$ is given. Comparison of optical and $21-\mathrm{cm}$ measurements of galaxian redshifts shows excellent agreement over the radical velocity range -400 to $+5200 \mathrm{~km} \mathrm{~s}^{-1}$. There is, however, a systematic difference between $21 \mathrm{~cm}$ and optical redshifts over the range $\sim 1200$ to $\sim 2400 \mathrm{~km} \mathrm{~s}^{-1}$ for optical values based on blue-sensitive spectra. The difference, $\sim 100 \mathrm{~km} \mathrm{~s}^{-1}$, is most likely due to blending of galaxian and night sky $H$ and $K$ absorption lines. The Hubble Constant is derived from a redshift-21 cm flux relation. Values in the range 78 to $109 \mathrm{~km} \mathrm{~s}^{-1} \mathrm{Mpc}^{-1}$ are derived. A value of $97 \mathrm{~km} \mathrm{~s}^{-1} \mathrm{Mpc}^{-1}$ is favored.
\end{abstract}

\section{Introduction}

Interstellar gas represents a significant constituent of most types of galaxies. As an example, one-fourth of the total mass of irregular-type systems is in the form of neutral atomic hydrogen. Assuming a normal abundance of helium - a point to be discussed in some detail below - the gaseous component reaches one-third of the total mass. In our galaxy, this number lies between 5 and $10 \%$.

Properly and completely to describe the gaseous component of a galaxy requires the evaluation of a distribution function giving the particle density of the various constituents, $N\left(p_{i}\right)$, in a volume element as a function of position within the galaxy. Additional descriptive information would include the motions of and the excitation condition within the volume element.

In the optical and radio domains, there are four physical processes that supply information to the observer on $N\left(p_{i}\right)$. These are:

(1) Emission: neutral and ionized gas;

(2) Absorption: gas and electrons;

(3) Dispersion: electrons;

(4) Scattering: electrons (also dust and gas).

* Operated by Associated Universities, Inc., under contract with the National Science Foundation. 
Additional 'probes' of interstellar matter are available through a study of cosmic rays, $\mathrm{X}$-rays, and $\gamma$-rays.

The presently available information does not allow us adequately to evaluate the above distribution function - even in our own galaxy. Nevertheless, a picture of the gaseous content of galaxies is slowly emerging for the nearer, and primarily, 'normal' systems. The complexity of the problem is indicated by the rich composition of the interstellar medium in our own Galaxy. Optical studies have identified interstellar lines of such atoms and molecules as sodium, calcium, titanium, iron, $\mathrm{CH}$, and $\mathrm{CN}$.

From studies at radio wavelengths we have thus far identified: neutral atomic hydrogen $(\mathrm{HI})$, the hydroxyl radical $(\mathrm{OH})$, water $\left(\mathrm{H}_{2} \mathrm{O}\right)$, ammonia $\left(\mathrm{NH}_{3}\right)$, formaldehyde $\left(\mathrm{H}_{2} \mathrm{CO}\right)$, hydrogen cyanide $(\mathrm{HCN})$, carbon monoxide $(\mathrm{CO})$, the $\mathrm{CN}$ radical, and cyano-acetylene $\left(\mathrm{HC}_{3} \mathrm{~N}\right)$. The $\mathrm{C}^{13}$ and $\mathrm{O}^{18}$ isotopes have been found in several of these substances. The presence of the hydrogen molecule $\left(\mathrm{H}_{2}\right)$, long suspected as being present in dark clouds, has been identified by Carruthers (1970) in a spectrum of the star $\xi$ Persei obtained in a rocket flight.

Ionized hydrogen (HiI) regions, which we assume to be representative of the interstellar medium in general, are excellent sources for studying a number of elements, especially nitrogen, oxygen, sulfur, and helium. We may expect our knowledge of the inventory of this chemical laboratory to continue to increase in the near future.

The electron density, $N_{e}$, of the interstellar medium shows immense variation, at least six orders of magnitude, from $\sim 10^{-2}$ to $\sim 10^{4} \mathrm{~cm}^{-3}$, the latter value holding for high-density $\mathrm{H}$ II regions. The low-electron density distribution has been described by Bridle and Venugopal (1969) in terms of a disk whose full thickness at half-intensity is $800 \mathrm{pc}$.

\section{The Chemical Composition of the Gas in Extragalactic Systems}

Information regarding the chemical composition of the interstellar material in extragalactic systems is almost wholly based on optically derived data. Qualitatively, the chemical composition of Hil regions in other galaxies, as evidenced by low and moderate dispersion spectra, is similar to that in our own Galaxy. Thus, similar lines and line ratios are seen in Orion and in extragalactic $\mathrm{H}$ II regions. This similarity was used by Hugh Johnson (1959) in the first determination of an extragalactic helium abundance; that of the 30 Doradus nebulae in the Large Magellanic Cloud. A summary of presently available determinations of the ratio $N\left(\mathrm{He}^{+}\right) / N\left(\mathrm{H}^{+}\right)$for different types of galaxies is displayed in Figure 1 . The data are from a variety of sources but are on a homogeneous system as far as recombination rates are concerned. The three filled circles at the right of the Sc category all refer to NGC 604, a giant Hil region in M33. Their scatter shows the range of uncertainty in such determinations.

The average value of this ratio for the extragalactic systems is 0.087 . For the six galactic Hil regions shown it is 0.092 .

The neutral helium component in HII regions may be allowed for through an ionization correction factor obtained from the abundance ratios of different ions of 


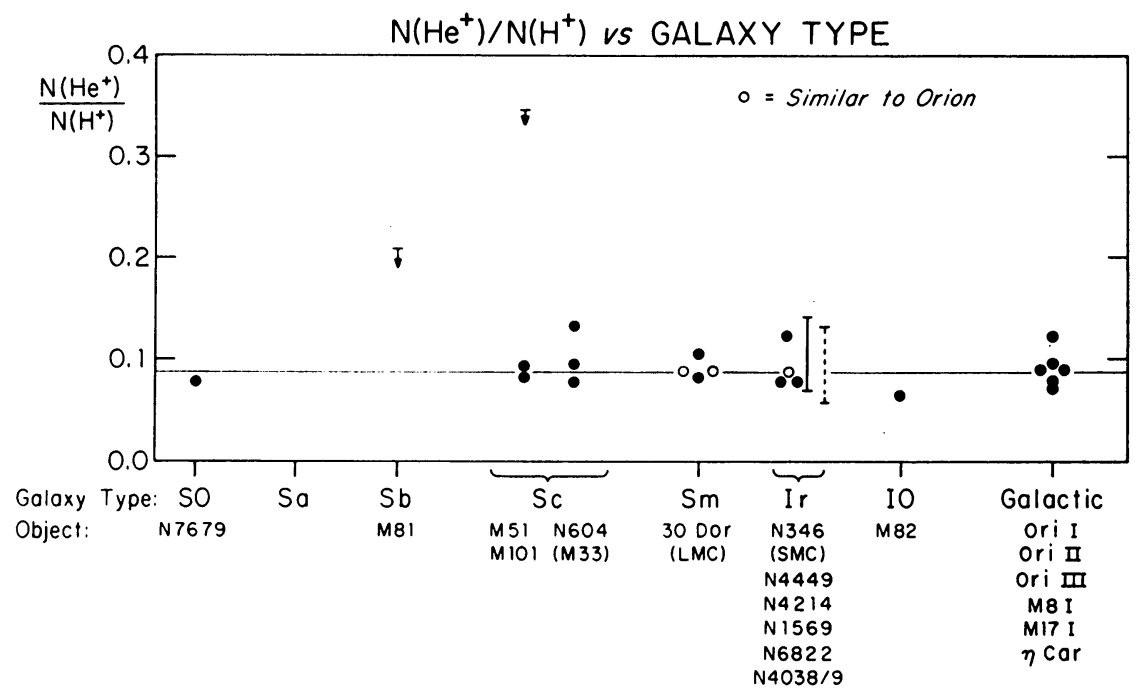

Fig. 1. The ratio $N\left(\mathrm{He}^{+}\right) / N\left(\mathrm{H}^{+}\right)$for different galaxy types and for the Galaxy. The Hil region or galaxy designation is indicated at the bottom of the figure. The three filled circles to the right of the Sc category refer to three different determinations for the same object, NGC 604 in M33. The solid bar in the irregular type refers to NGC 4214 and represents the limits applied by Mathis (1965) to this ratio. The dashed bar shows the range of $N\left(\mathrm{He}^{+}\right) / N\left(\mathrm{H}^{+}\right)$found for different $\mathrm{HII}$ regions in the peculiar galaxy NGC 4038/9 by Rubin et al. (1970).

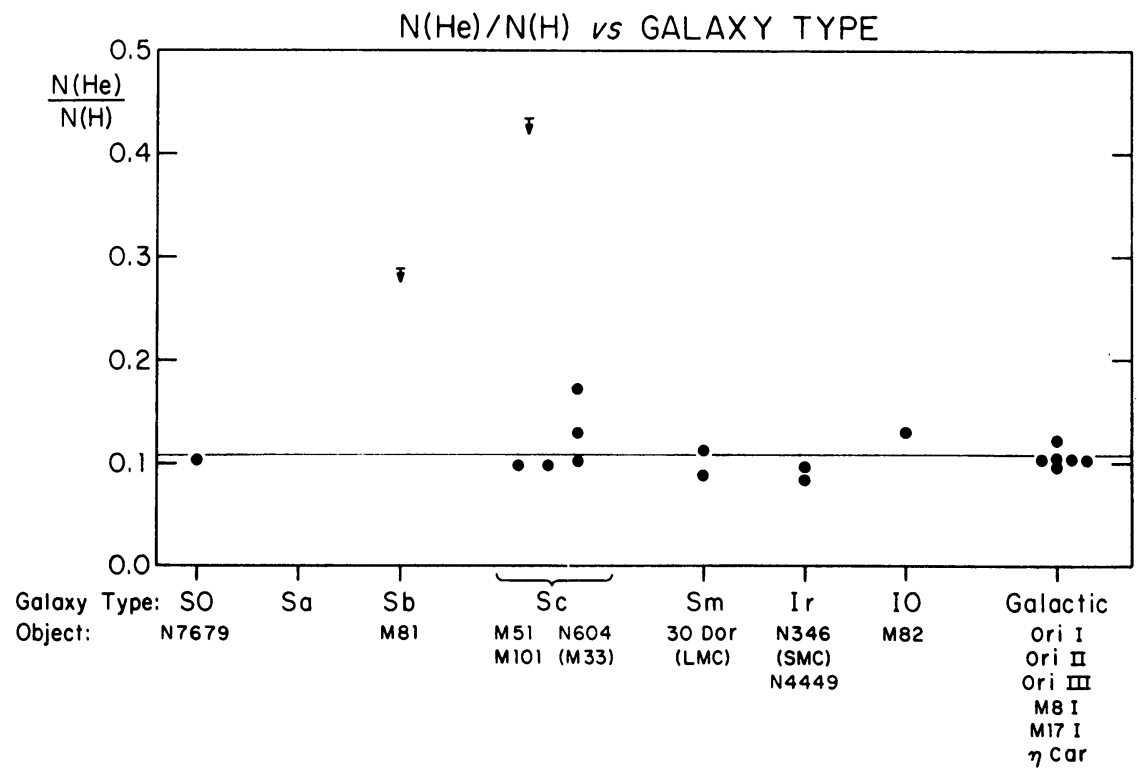

Fig. 2. The ratio $N(\mathrm{He}) / N(\mathrm{H})$ for different galaxy types and for the Galaxy. The three filled circles to the right of the Sc category refer to three different determinations for the same object, NGC 604 in M33. The Hil region or galaxy designation is indicated at the bottom of the figure. 
a particular element such as oxygen or sulphur (Peimbert and Costero, 1969). Figure 2 displays the ratio $N(\mathrm{He}) / N(\mathrm{H})$ as a function of structural type. Most of these data are from Peimbert and Spinrad (1970). The large scatter for NGC 604 is again evident. The average value of the helium-to-hydrogen ratio for the extragalactic systems is 0.11 ; for the galactic $\mathrm{H}$ il regions it is the same. This constancy of the helium-to-hydrogen ratio in various spirals and irregular-type systems, regardless of structural type, has been recognized for some time. The more recent data strengthen this conclusion. I shall return to this subject later after discussing the hydrogen content of galaxies.

The question of a variation of helium abundance with distance from the center of a galaxy was first discussed by Schmidt (1962). He obtained spectra of three H II regions in Andromeda at true distances from the center of $25^{\prime}, 70^{\prime}$, and $89^{\prime}$ and found that the ratio of the $\mathrm{D} 3$ line of helium to $\mathrm{H} \beta$ was constant to within a factor of two, which was the uncertainty he assigned to his measurements. Schmidt concluded that the helium-to-hydrogen ratio was essentially constant over this range of distances. More recent and more extensive work by Rubin (1970, unpublished) supports this earlier result. She finds no systematic variation with radial distance of the helium-tohydrogen ratio in M31. Searle (1970) reaches a similar conclusion for Sc-type galaxies. This absence of a correlation of helium abundance with radial distance is indirectly supported by the material presented in Figures 1 and 2 since the $\mathrm{H}$ II regions involved in the measurements for the various galaxies are at different relative distances from the center.

There does appear to be a systematic variation of nitrogen with respect to hydrogen as a function of radial distance. The variation of $[\mathrm{N} I \mathrm{II} / \mathrm{H} \alpha$ within a galaxy and among different galaxies has been described by the Burbidges $(1962,1965)$. Initially, these variations were thought as being possibly due to excitation effects (Burbidge et al., 1963). However, Peimbert's (1968) observations of the [OI], [OII], and [O III] lines, in addition to $[\mathrm{NII}]$ and $\mathrm{H} \alpha$ yield the conclusion that the variations are caused by an abundance effect. For the nuclear regions of M51 and M81, Peimbert found, by adopting a solar oxygen-to-hydrogen ratio, an excess of nitrogen of from two to six times the solar value.

Searle (1970) and Rubin (1970) find similar abundance variations in other galaxies.

In summary, we conclude that the helium-to-hydrogen ratio is constant in galaxies of structural types Ir through S0 and is also constant across the disks of these galaxies although there may be local variations. This is not the case for the nitrogen-tohydrogen ratio which does vary with structural type as well as radial distance within a galaxy.

\section{The Distribution of Neutral Hydrogen Within Galaxies}

The most abundant element, hydrogen, cooperates to a surprising extent by allowing us to study it in a relatively direct and easy manner in both its neutral and excited states. The recombination lines of excited hydrogen can be studied at both optical and radio wavelengths; neutral atomic hydrogen is measurable through its line 
radiation at $21 \mathrm{~cm}$. With this latter probe, we are able to map the distribution of neutral hydrogen; measure its total content in a galaxy; obtain information on the dynamics within a galaxy and hence its total mass; and finally we can obtain an accurate measure of the systemic velocity of a galaxy.

The material which I will discuss is based on measurements made at a number of observatories, and I shall not attempt to credit the many people involved except when I speak of specific galaxies. Details may be found in a survey by Roberts (1969) of the data available through mid-1969.

For the larger galaxies, filled aperture observations supply sufficient relative resolution to outline the gross features of the Hi distribution. These features may be conveniently categorized as: (1) Main body distribution; (2) Extent; (3) Companions; (4) Warp or bending of the plane; (5) Bridges.

Some of these may be interrelated but at present we do not have sufficient information for a large enough sample to do much more than describe such features.

$21-\mathrm{cm}$ studies of our Galaxy are a convenient starting point. There are many interesting features in the galactic hydrogen distribution, e.g. the arc-shaped hydrogen concentrations termed 'arms', the high velocity clouds, the extreme flatness of the plane of $\mathrm{Hr}$ interior to the Sun, and the bending or warp of the plane exterior to the Sun. For comparison with other galaxies, we shall consider only the gross features of the galactic HI distribution. These are: (1) The deficiency of $\mathrm{HI}_{\mathrm{I}}$ in the central region, i.e., a 'ring' of $\mathrm{HI}$; (2) The percentage of the total mass in the form of neutral atomic hydrogen; and (3) The bending of the HI plane in the outer regions of our Galaxy.

The term 'ring' is intended merely as a convenience in describing the average radial variation of the hydrogen surface density (projected onto the plane of the galaxy). Burke (1967) has described the ring as "a disk with a hole in the middle". This is a more descriptive (and more lengthy) phrase since the ring is quite thick in the radial direction. In our Galaxy the radius at maximum density is about $10 \mathrm{kpc}$ and its full width at half intensity is also about $10 \mathrm{kpc}$. Such a ring-like distribution is not unique to our Galaxy. The half dozen spiral galaxies thus far studied with high enough relative resolution all show a similar distribution.

An interesting and unexplained feature of this ring of neutral hydrogen is its position relative to the location of the principal concentrations of ionized hydrogen, that is, the optical spiral arms. In our Galaxy the giant $\mathrm{H}$ II regions, as defined by radio recombination line observations, lie primarily interior to this ring at a distance of $\sim 4$ to $6 \mathrm{kpc}$ (Reifenstein et al., 1970). The distribution of the thermal continuum radiation also implies a similar central concentration. Presumably, both of these data refer to a prominent spiral arm of our Galaxy. Figure 3, taken from Professor Oort's (1965) Invited Discourse at the Hamburg IAU, shows these features quite clearly.

A similar placing of $\mathrm{HI}$ and $\mathrm{HII}_{\mathrm{II}}$ concentrations is found in other spiral galaxies. The prominent, optically-defined spiral features lie interior to the HI ring. An outstanding exception is M31. In this Sb-type galaxy, the optical arms are embedded in the HI ring. The HI ring in M31 is clearly evident in the isometric projection of 


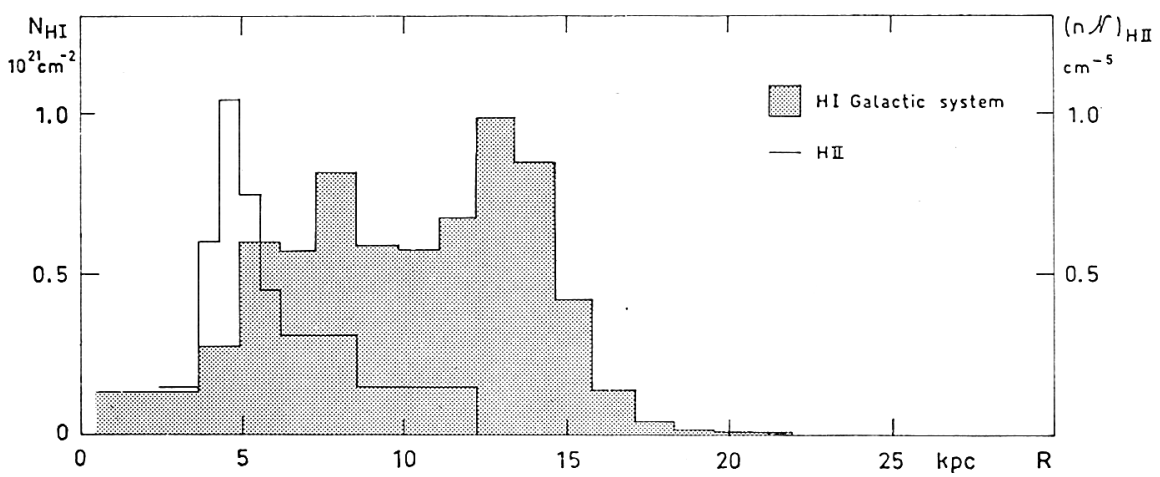

Fig. 3. The radial distribution of neutral and ionized hydrogen in our Galaxy. This figure is from Oort (1965).

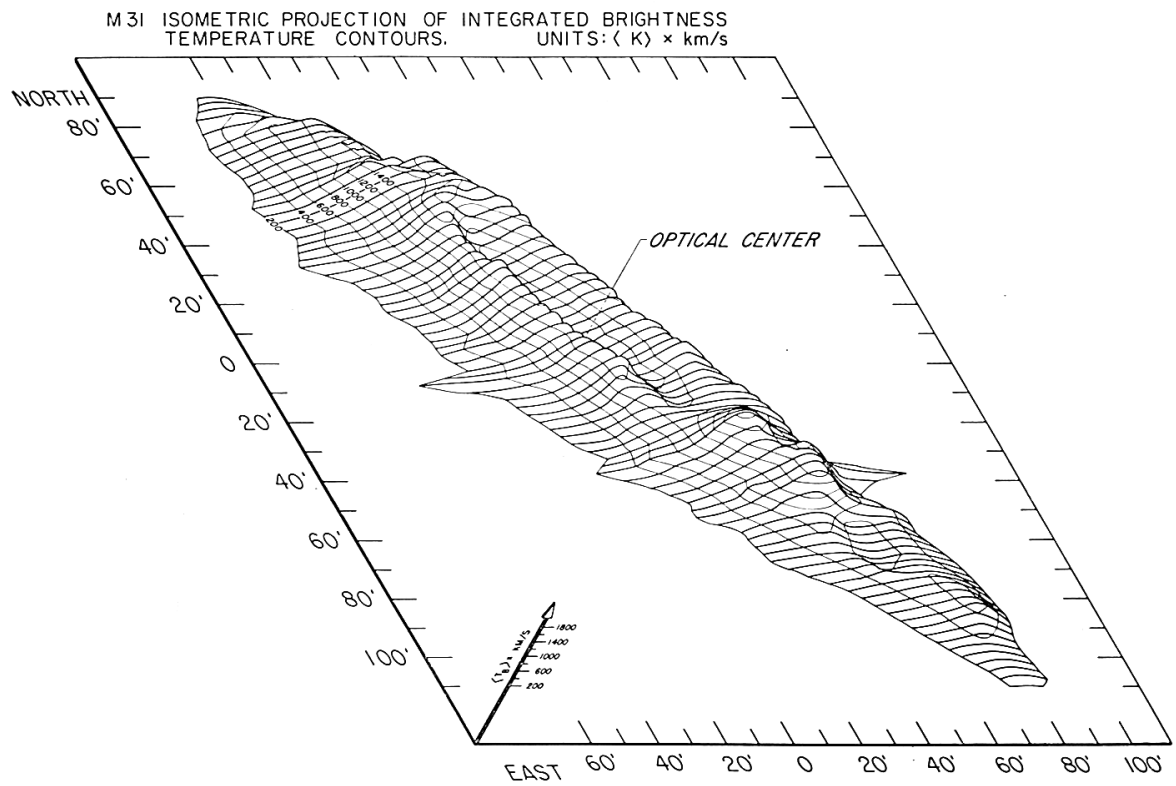

Fig. 4. An isometric projection of 21-cm integrated brightness temperature contours for M31. The lack of a central concentration of neutral hydrogen is clearly evident. These data were obtained with the 300-foot telescope (beamwidth $=10^{\prime}$ ).

integrated brightness temperature contours shown in Figure 4. M31 is the only $\mathrm{Sb}$ system in our rather meagre sample and it is obviously premature to attempt to relate the relative location of neutral and ionized hydrogen to structural type. However, we do have several irregular-type galaxies mapped with sufficient resolution and they do not exhibit a deficiency of $\mathrm{HI}_{\mathrm{I}}$ in their central regions. This additional datum does suggest a relation between the region of star formation and the maximum surface density of $\mathrm{HI}$ with type, but more information on earlier-type galaxies is required before this conclusion can be considered well established. 
Several clarifying points should be made regarding these features. The hydrogen ring is really defined by the observational quantity of integrated (over velocity), beamaveraged brightness temperature. A minimum in the center of the ring could be due to an observational effect in which much of the $\mathrm{HI}_{\mathrm{I}}$ is concentrated into small regions of high optical depth. Beam-dilution would then give the appearance of a minimum of HI. Another possibility is that much of the hydrogen is in the form of molecular hydrogen. A third possibility is that the hydrogen is ionized. Finally, there may indeed be a relative deficiency of the neutral hydrogen in the central regions of later-type spirals. Regardless of the correct explanation we cannot escape the fact that there is an over-all variation in spirals in the form of amount of hydrogen with respect to distance from the center.

The ring itself has been described as a smooth circular feature. This is a great simplification forced by lack of sufficient resolving power. The ring need not be circular and surely has a large fluctuation in its density structure. Further, the concentration of ionized hydrogen regions interior to the maximum of the ring does not imply that there is no neutral hydrogen in these regions. Rather, we may expect local concentrations of neutral hydrogen near and about $\mathrm{HII}$ regions, as shown by Orion in our Galaxy (Menon, 1958; C. Gordon, 1970) and the Hil regions in the Large Magellanic Clouds (McGee, 1964; McGee and Milton, 1966).

Although there are some HII regions in the ring, an obvious question is: Why haven't the spiral arms formed in this higher density region? Lin (1970) has suggested

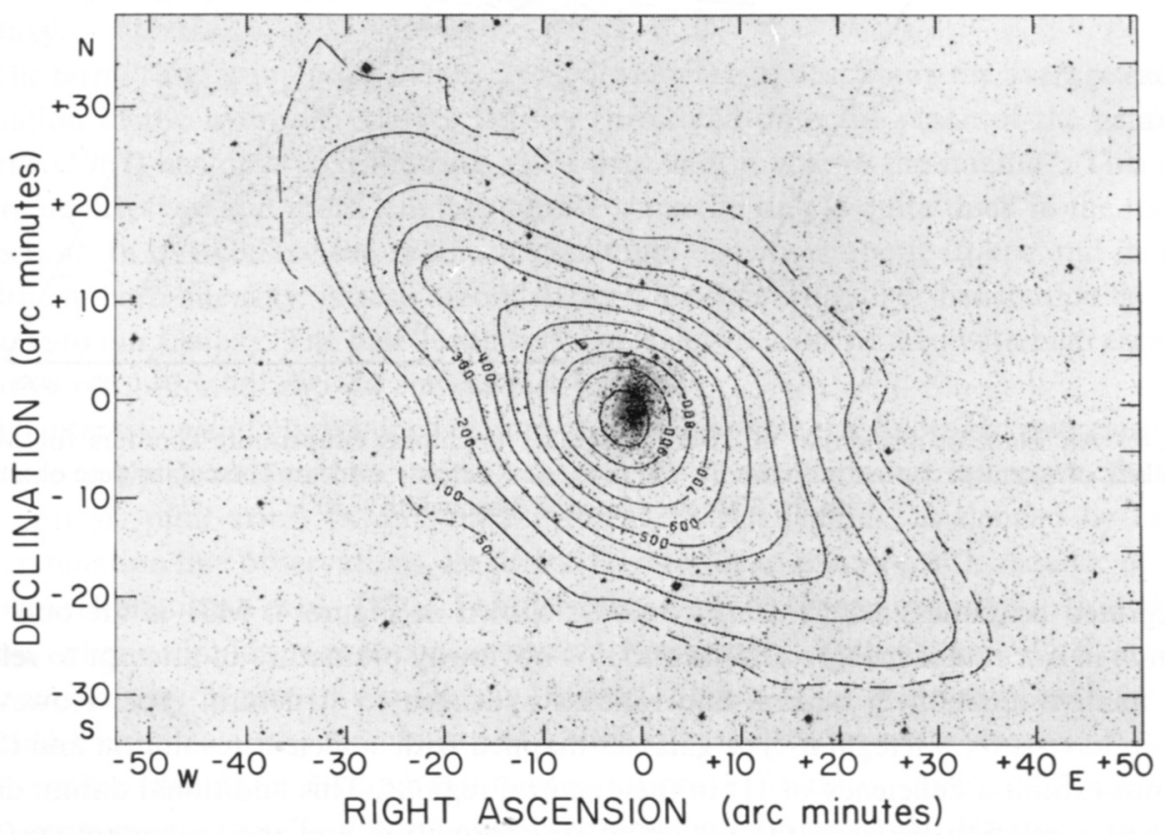

Fig. 5. Contours of $21-\mathrm{cm}$ integrated brightness temperature superposed on a photograph of the irregular-type galaxy NGC 6822. These data were obtained with the 300 -foot telescope (beam width $=10^{\prime}$ ). 


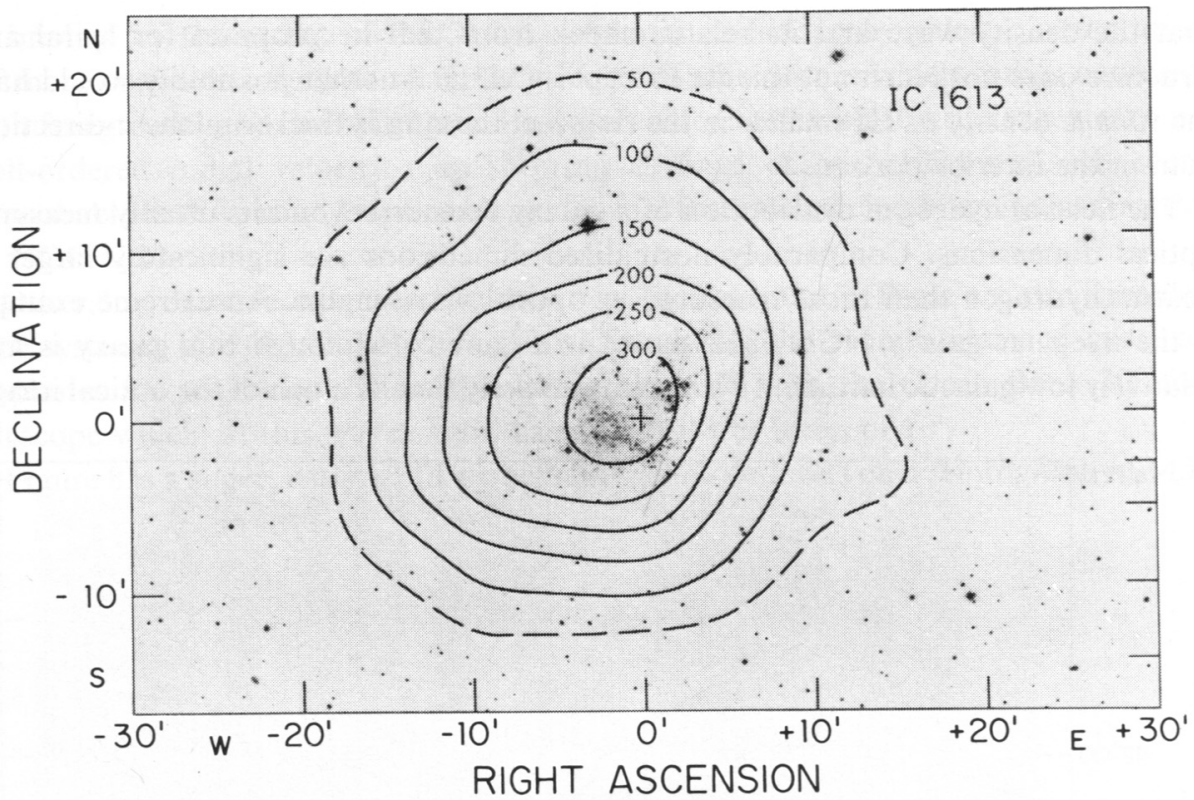

Fig. 6. Contours of 21-cm integrated brightness temperature superposed on a photograph of the irregular-type galaxy IC 1613 . These data were obtained with the 300 -foot telescope (beam width $=10^{\prime}$ ).

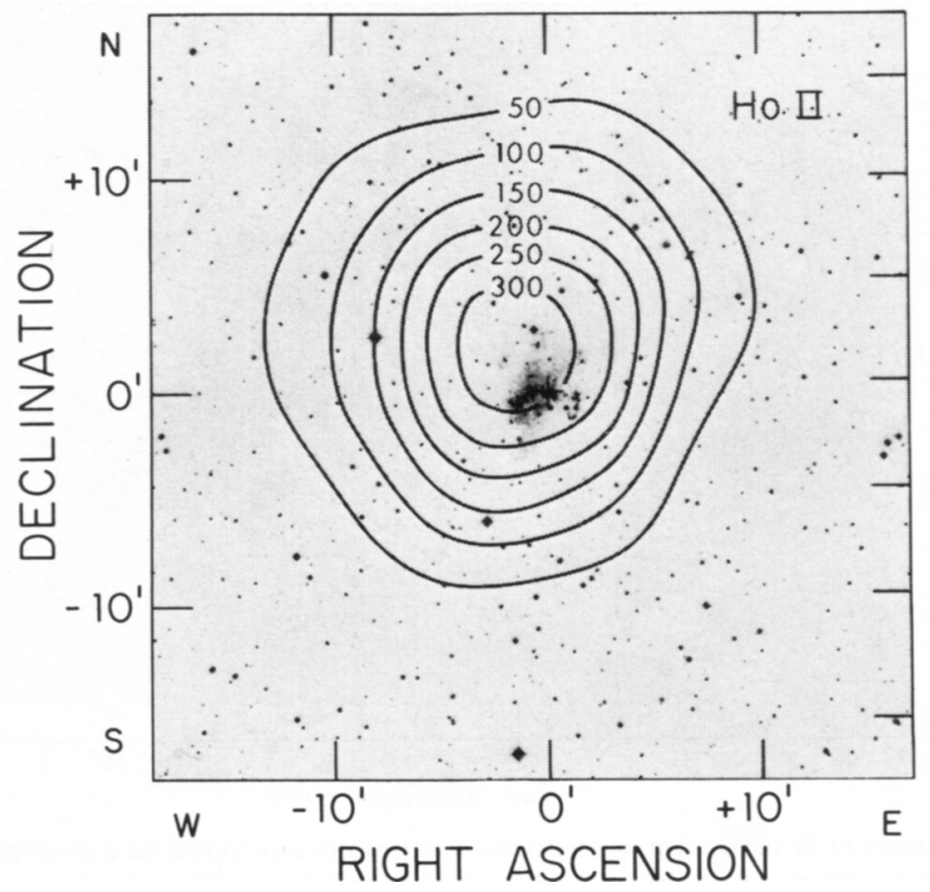

Fig. 7. Contours of $21-\mathrm{cm}$ integrated brightness temperature superposed on a photograph of the irregular-type galaxy Hoir. These data were obtained with the 300 -foot telescope (beam width $=10^{\prime}$ ). 
that the density wave and its related shock front that he proposes for spiral arm structure does not reach out this far (except in M31). Another possibility would have the volume density of $\mathrm{HI}$ smaller in the ring (i.e. the ring is thicker in the $z$-direction) than in the interior portions.

The neutral hydrogen distribution of a galaxy extends beyond its usually measured optical dimensions. Comparably normalized dimensions are significantly larger in neutral hydrogen than those measured at optical wavelengths. An extreme example is the irregular galaxy NGC 6822 shown in Figure 5. Although this galaxy is at a relatively low galactic latitude, $18^{\circ}$, it seems unlikely that as much of the optical image,

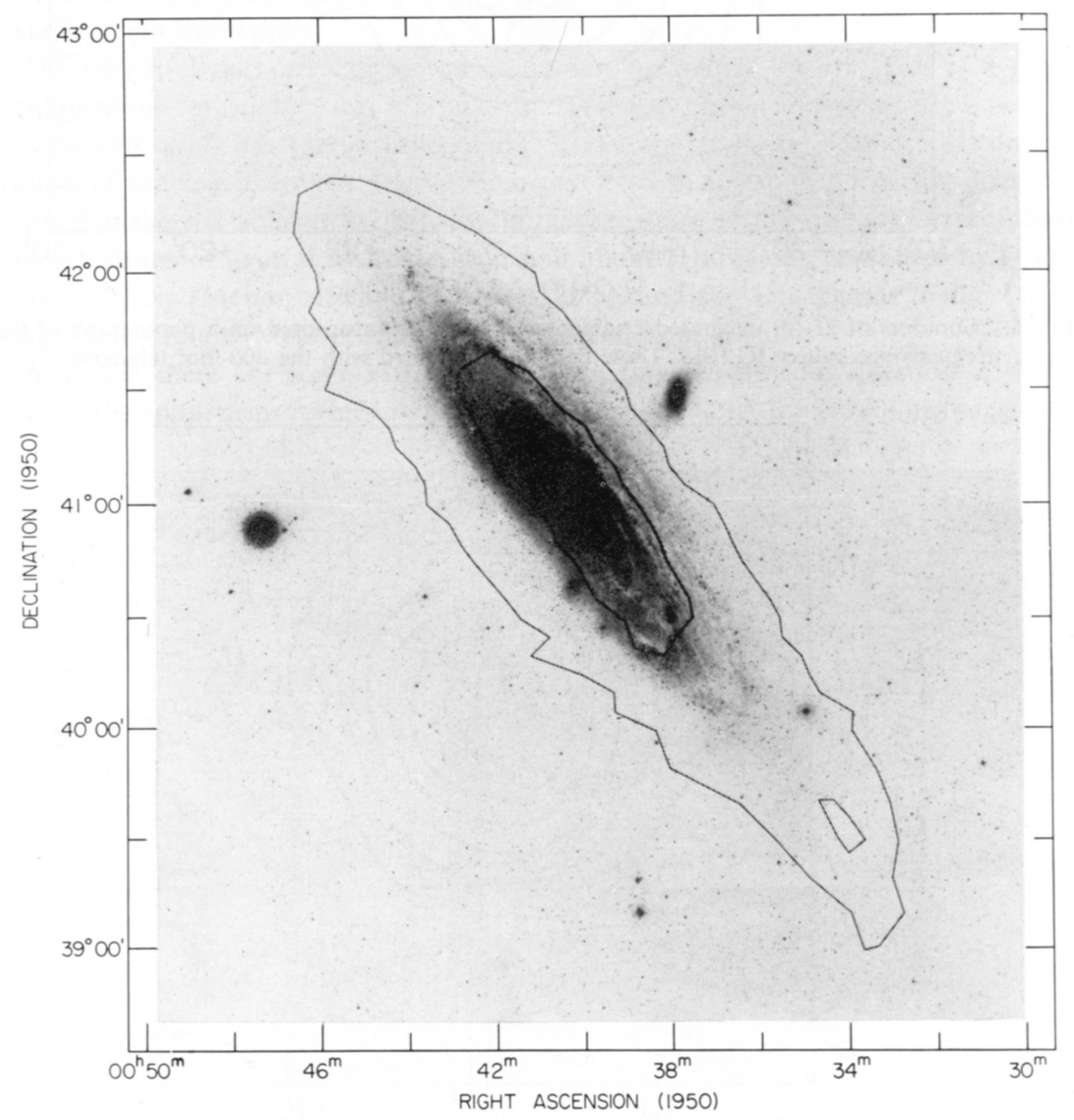

Fig. 8. Contours of $21-\mathrm{cm}$ integrated brightness temperature superposed on a photograph of $\mathrm{M} 31$. The inner, heavy contour represents the peak brightness temperature. The outer contour corresponds to $200 \mathrm{~K} \mathrm{~km} \mathrm{~s}^{-1}$ and is from $\frac{1}{6}$ to $\frac{1}{8}$ the peak contour. The south-preceding feature is represented in the lower right by a contour level of $600 \mathrm{~K} \mathrm{~km} \mathrm{~s}^{-1}$. These data were obtained with the 300-foot telescope (beam width $=10^{\prime}$ ). 
as implied by the extent of the $21-\mathrm{cm}$ isophotes, is hidden by galactic obscuration. Of more concern is the low systemic velocity of this system which causes confusion with foreground galactic hydrogen. However, associated with this hydrogen map is a well-ordered radial velocity map showing a range of over $100 \mathrm{~km} \mathrm{~s}^{-1}$. It seems improbable that we would have such an unusual foreground cloud just in the direction of NGC 6822.

Other, less extreme, examples are shown in Figures 6 and 7. These are also irregulartype systems, IC 1613 and HoII. For all three cases, the beam-broadened hydrogen isophotes are as shown. These data were obtained with the Green Bank 300-foot telescope which, at this wavelength, has a half-power beam of $10^{\prime}$.

Figure 8 is a superposition of 2 principal Hi contour levels on a photograph of M31.

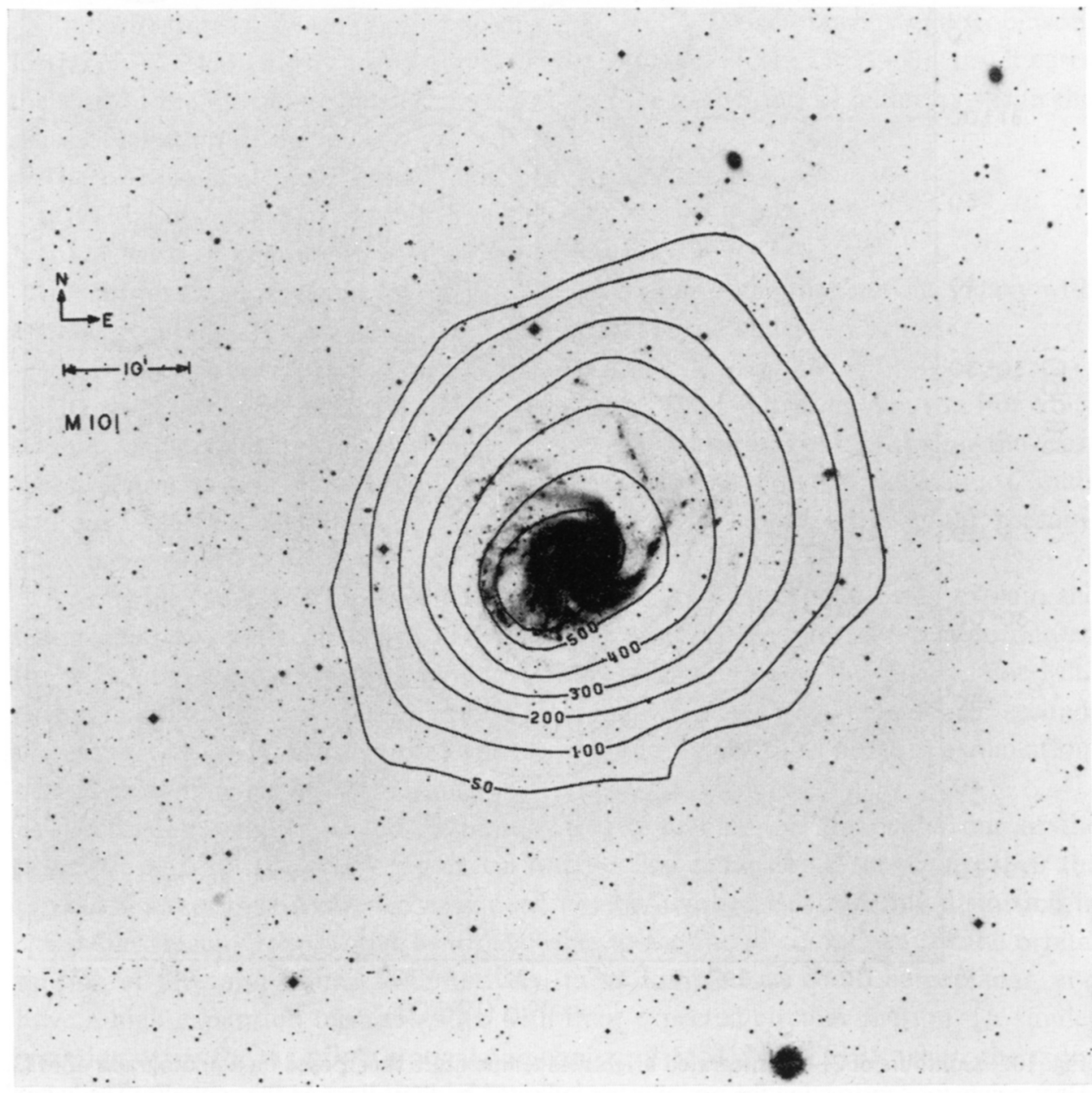

Fig. 9. Contours of 21-cm integrated brightness temperature superposed on a photograph of M101. The asymmetrical $\mathrm{HI}_{\mathrm{I}}$ distribution to the northeast is clearly evident. These data were obtained with the 300-foot telescope (beam width $=10^{\prime}$ ). 
The inner contour locates the peak integrated brightness temperature. The outer contour is $\frac{1}{6}$ to $\frac{1}{8}$ of the average peak level. These data are also broadened by a $10^{\prime}$ beam. The south-preceding feature first delineated by Burke et al. (1963) can be seen at one end of the major axis. On this relative-resolution scale it stands out almost as a separate feature. For more distant galaxies such a feature would appear only as a smooth extension of the general contours. A possible example of this is shown in

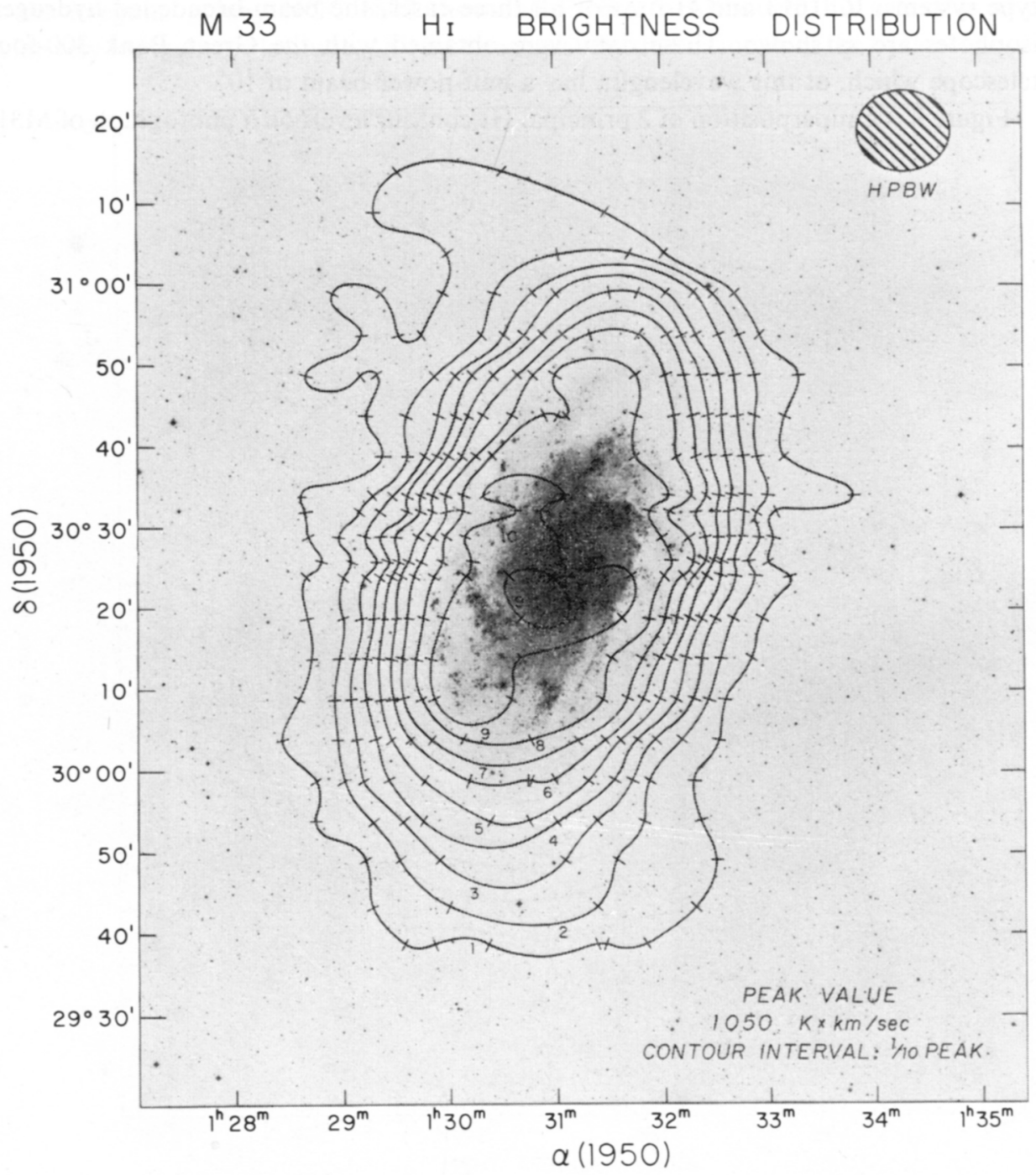

Fig. 10. Contours of $21-\mathrm{cm}$ integrated brightness temperature superposed on a photograph of M33. Data points for constructing the contours are indicated by short lines on the contours. The 'wings' to the northwest (top left) and southeast (bottom right) are clearly evident. These data were obtained by $\mathrm{K}$. Gordon (1970) using the 300 -foot telescope. The signal-to-noise ratio has been improved by smoothing. The resultant beam-size is shown in the figure. 
Figure 9, which is a map of hydrogen contours superimposed on a photograph of M101. The asymmetrical distortion to the north-east may represent beam-smearing of an adjacent hydrogen companion.

Figure 10 is of M33 and shows the relation of the optical image and hydrogen isophotes as derived from 300 -foot observations. These data were obtained by K. Gordon (1970). Gordon finds that to comparable levels, the hydrogen extent is approximately twice that given by star counts, B photometry, and the distribution of Hil regions.

There is another feature in M33 to which attention is directed. This is the significant deviation of the outer isophotes from the optical major axis. A line connecting these outer features is displaced from the major axis position angle in the direction of rotation of $\mathbf{M} 33$. The radial velocity map for $\mathbf{M} 33$ shows a displacement in the same sense as the $\mathrm{HI}$ contours.

Similar features are seen in other spirals, e.g., NGC 300 (Shobbrook and Robinson, 1967), NGC 2403 (Burns and Roberts, 1970), NGC 5236 (Lewis, 1968). In all cases the shift of the kinematic and HI axes with respect to the optical major axis is in the sense of rotation of the galaxy.

There are several possible explanations for such features:

(a) Tidal distortion by neighboring galaxies;

(b) A warp or bending of the plane;

(c) A non-axisymmetric expansion of the hydrogen in the plane of the galaxy with a stable region on the outskirts of the galaxy where the hydrogen collects;

(d) Hydrogen companions.

The problems of invoking tidal distortion for our Galaxy are well known. For M33, as an example, the situation is even more severe if one wishes to use M31 as the most massive, nearby interacting system (K. Gordon, 1970). As with the Magellanic Clouds and our Galaxy, a near encounter in the orbital motion of M31-M33 could, presumably, be invoked.

A warp in the plane similar to that in our Galaxy will qualitatively explain the features. However, the distortion in the radial velocity field is too great to be accounted for without requiring a large radial streaming in the plane of the galaxy. For M33 such a non-axisymmetric radial motion term is several tens of kilometers per second, increasing outwards. These remarks obviously hold for the third possible explanation, of non-axisymmetric radial motion.

The final possibility would require hydrogen companions in orbits out of the principal plane of the primary galactic system. The expansion term required for the physically connected hydrogen case need not be invoked. Instead, the distortion in the radial velocity map would be attributable to the radial component of the orbital motion of the companions. Alternatively, these companions could be coplanar, and have a high expansion term together with their expected circular motion. [A similar situation exists for the bright optical companion of M51, NGC 5195, where the radial velocity difference of these two systems can only be explained by an expansion term or by an orbit out of the plane of M51 (Roberts and Warren, 1970).]

Of the various explanations, the hydrogen companions appears the most attractive. 
In addition, warps in the Hi plane of the primary system may also exist and could, possibly, be attributed to these companions.

'Bridges' are the final aspect in the list of hydrogen distribution features. The first described example of such a feature is the link between the Large and Small Magellanic Clouds (Hindman et al., 1963). This link has been studied in some detail by Turner (1970). There are also suggestions that a link exists between our Galaxy and the Magellanic Clouds. Another example is the bridge between NGC 4631 and 4656 (Roberts, 1968; Weliachew, 1969).

Yet another striking example of such hydrogen bridges is found in the triple

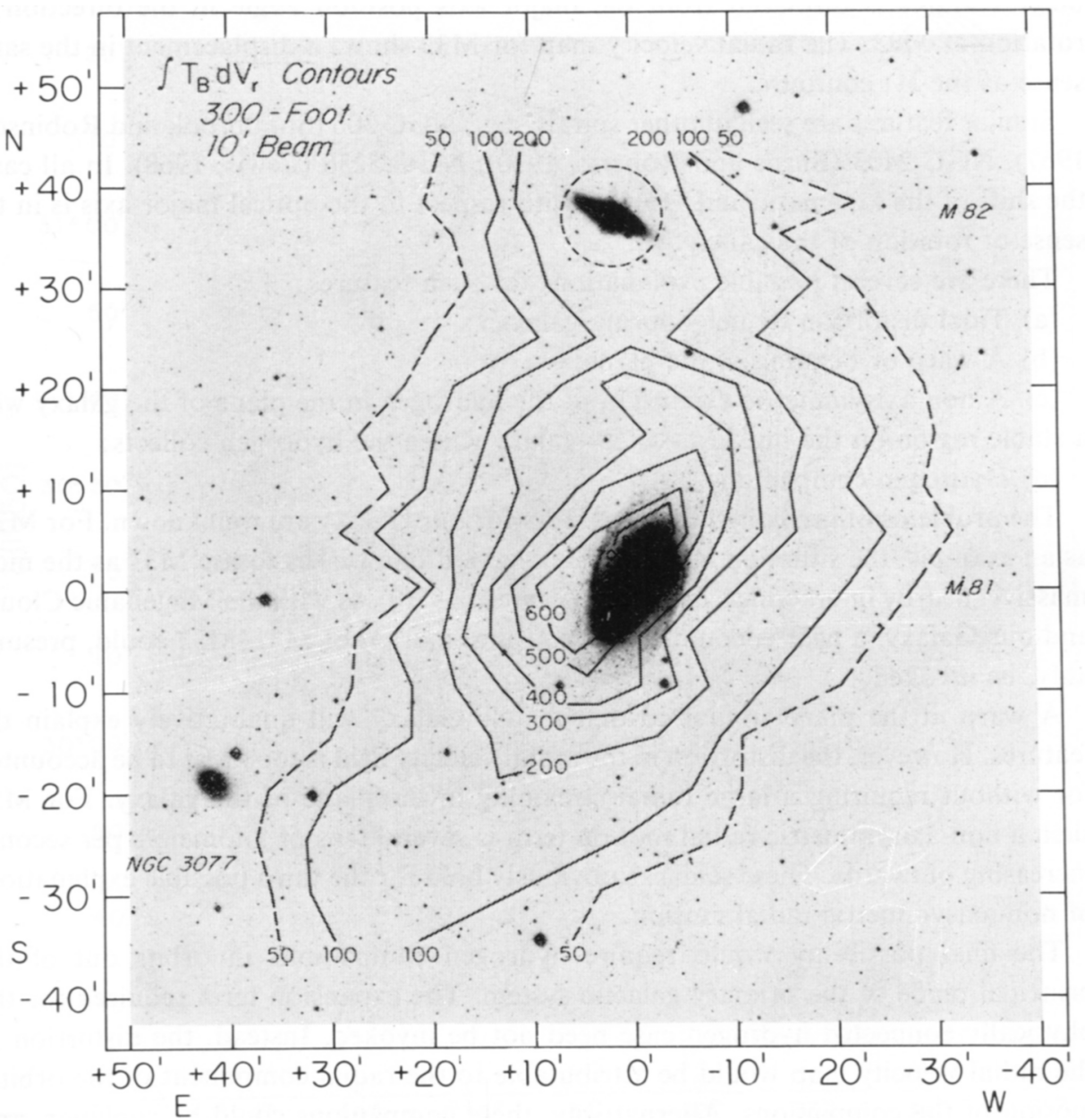

Fig. 11. Contours of $21-\mathrm{cm}$ integrated brightness temperature in the area of the triple system M81-M82-NGC 3077. The photograph is from the red sensitive National Geographic Sky Survey Atlas. Data from the region of M82 (dashed circle), a continuum source, are omitted because of possible gain imbalance between signal and reference frequencies. The integration is over all velocities. Because of low systemic velocity of M81 there is some confusion with foreground galactic hydrogen in the region of M81. The contours include an extrapolation over this velocity range. The other regions are free of confusion; see the velocity map in Figure 13. 
system of M81-M82-NGC 3077. (A more appropriate term in this case might be hydrogen streamers.) Figure 11 shows the beam-averaged hydrogen distribution as seen with the $10^{\prime}$ beam of the 300 -foot telescope. The hydrogen associated with M81 is clearly distorted in the directions of M82 and NGC 3077. These data were obtained

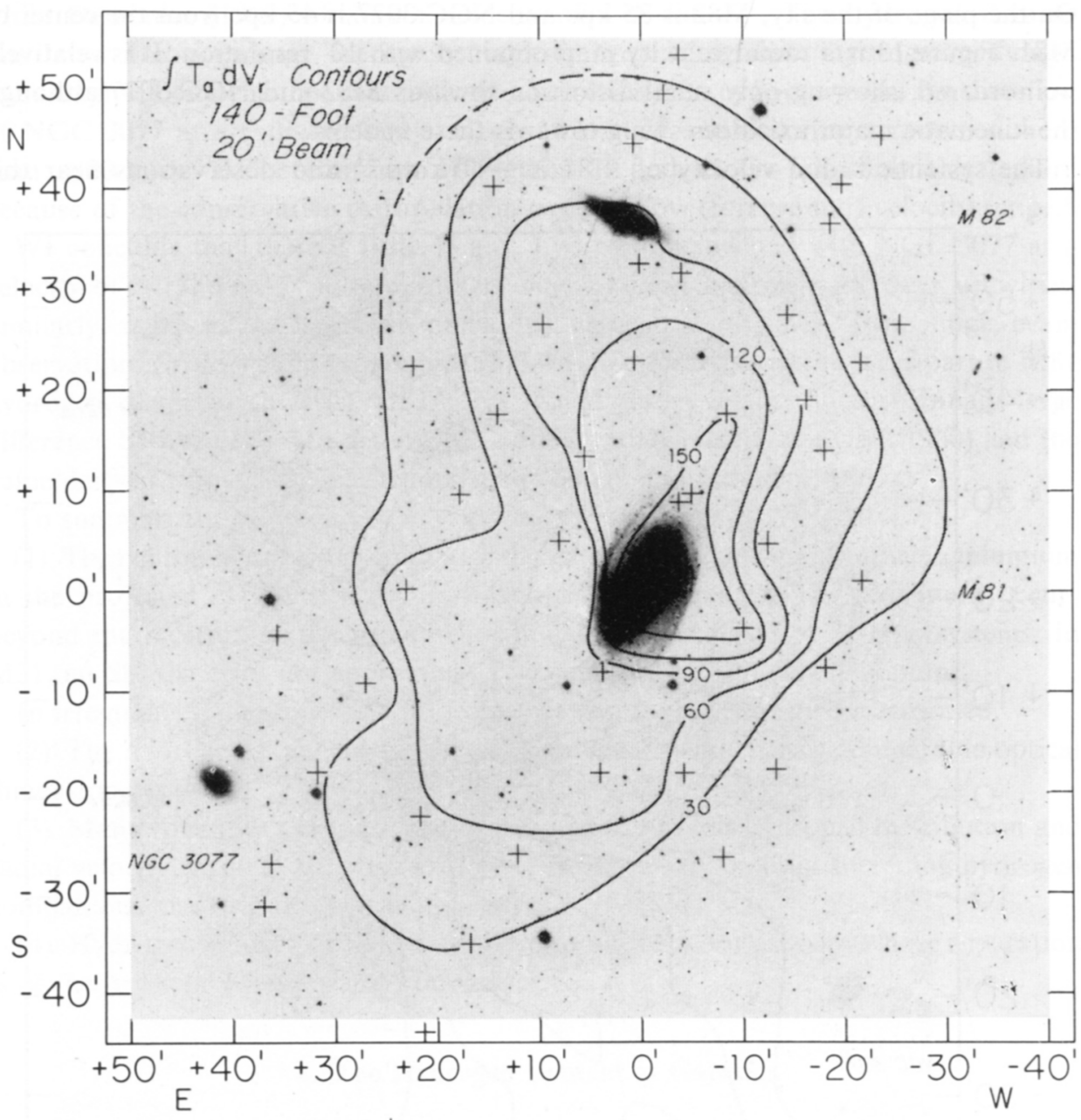

$++$

Fig. 12. Contours of $21-\mathrm{cm}$ integrated antenna temperature in the of area the triple system M81-M82-NGC 3077. The photograph is from the red sensitive National Geographic Sky Survey Atlas. An autocorrelation receiver was used for these observations and the data near M82 are therefore free of gain imbalance due to the continuum radiation from M82. The points observed are indicated by plus signs. The integration is over all velocities. Because of the low systemic velocity of M81 there is some confusion with foreground galactic hydrogen in the region of M81. The contours include an extrapolation over this velocity range. The other regions are free of confusion; see the velocity map in Figure 13. 
with a multichannel receiver and the observations on M82, a radio continuum source, are not included because of possible gain imbalance between the signal and reference frequencies. Figure 12 is a similar map, obtained with the $20^{\prime}$ beam of the 140 -foot telescope. An autocorrelation receiver, which does not suffer from gain imbalance problems, was used. We see a hydrogen distribution similar to the 300 -foot data. On the plane of the sky, M82 is $35 \mathrm{kpc}$ and NGC 3077 is $45 \mathrm{kpc}$ from the center of M81. Figure 13 is a radial velocity map obtained with $10^{\prime}$ resolution. It is relatively well-ordered, showing only small distortion towards M82 and NGC 3077 although the kinematic major axis does swing towards these systems.

The systemic radial velocity of M81 is $-40 \mathrm{~km} \mathrm{~s}^{-1}$ and observations near this

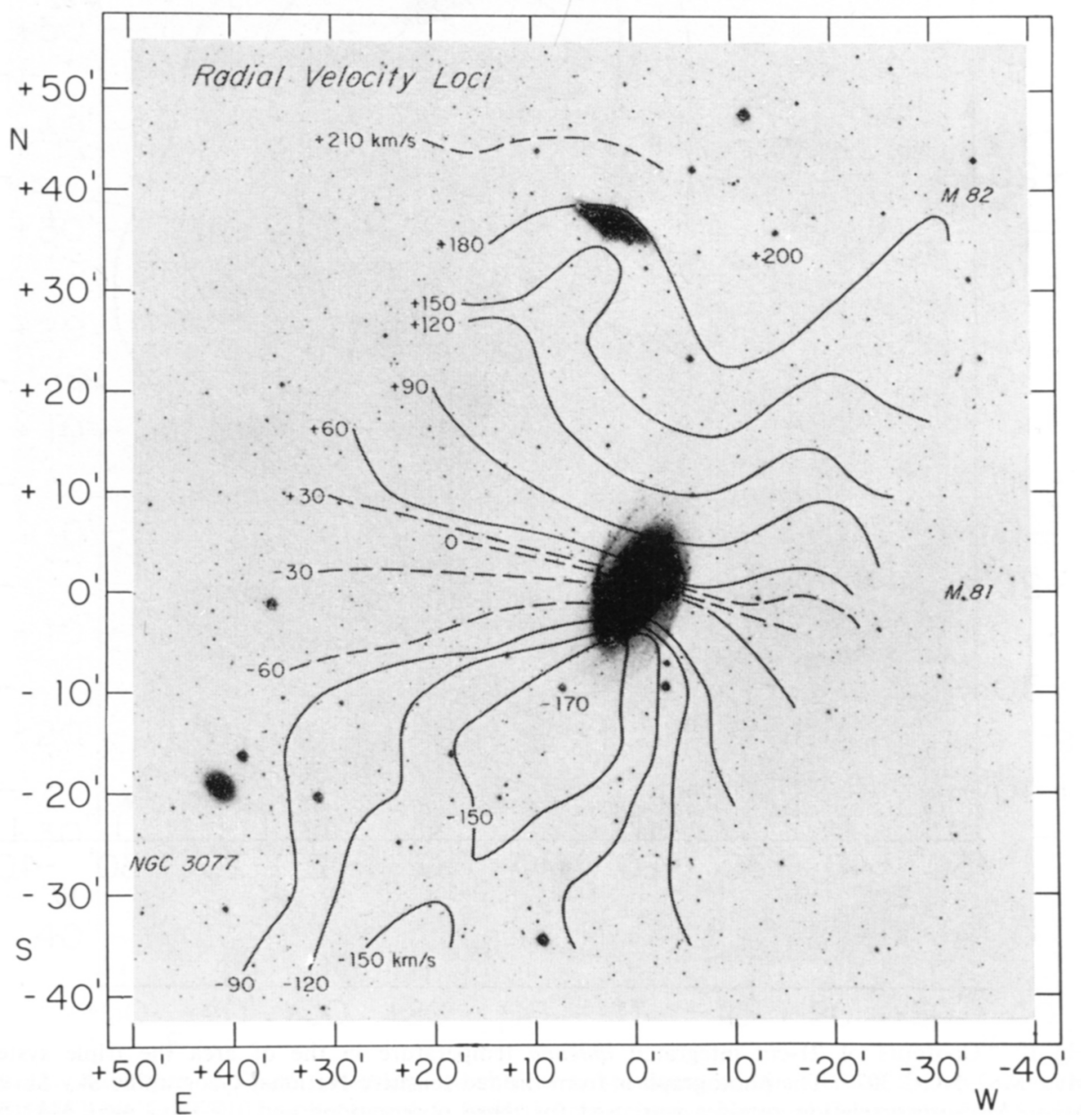

Fig. 13. A radial velocity map (loci of constant radial velocity) in the area of the triple system M81-M82-NGC 3077. The photograph is from the red sensitive National Geographic Sky Survey Atlas. These loci were constructed from the midpoints of velocity profiles obtained with the 300-foot telescope (beam width $=10^{\prime}$ ). The loci near zero velocity are confused by foreground galactic hydrogen and are therefore uncertain. 
velocity are seriously affected by foreground galactic hydrogen. For this reason, the low velocity loci in Figure 13 are uncertain. However, the optically-measured velocity of M82 is well separated from galactic hydrogen and the distorted hydrogen toward this system is free of foreground contamination. The situation is less clear for NGC 3077. Humason et al. (1956) give a velocity of $-158 \mathrm{~km} \mathrm{~s}^{-1}$ for this system while Demoulin (1969) obtains a velocity of $-41 \mathrm{~km} \mathrm{~s}^{-1}$. The hydrogen data in this latter velocity range are again confused with foreground hydrogen although individual velocity channel data indicate a concentration of hydrogen in the direction of NGC 3077 at a radial velocity of $\sim+10 \mathrm{~km} \mathrm{~s}^{-1}$ and covering a range of more than $\pm 30 \mathrm{~km} \mathrm{~s}^{-1}$. The contours of Figures 11 and 12 do not show this feature because of the conservative extrapolation over the low (foreground) velocity range.*

We conclude that there is little, if any, hydrogen associated with NGC 3077 at a velocity of $-158 \mathrm{~km} \mathrm{~s}^{-1}$ although there may be some hydrogen at lower velocities. Similarly, much of the hydrogen previously associated with M82 from single-beam observations (Volders and Högbom, 1961) would appear to refer in large part to M81 hydrogen distorted towards M82. This would also explain the surprisingly large difference between the well-determined optical radial velocity (Mayall, 1960) and the value derived from $21-\mathrm{cm}$ measurements (Volders and Högbom, 1961).

To summarize:

(1) The neutral hydrogen in spirals is not centrally concentrated. Rather, a minimum in the projected HI density occurs near the optical center. The maximum occurs beyond the location of the prominent optical spiral features in Sc-type systems. In $\mathrm{M} 31$, an $\mathrm{Sb}$, the arms are approximately coincident with the $\mathrm{HI}$ maximum.

In irregular-type galaxies the projected $\mathrm{HI}$ density is centrally concentrated.

(2) The hydrogen extent of a galaxy is significantly larger than comparable optical dimensions.

(3) Many (possibly all) spirals show distortions in their hydrogen distribution and radial velocity map. There are a variety of possible explanations including hydrogen companions and/or a warp in the plane.

(4) Hydrogen bridges or links have been found between galaxies whose separation is of the order of a few tens of kiloparsecs.

\section{The Hydrogen Content of Galaxies}

One of the clearest correlations with structural type of a quantitatively measured parameter is obtained with the neutral hydrogen content of a galaxy. Using photographic luminosity as a normalizing factor, Heidmann (1961) found such a correlation in the sense that the ratio $M_{H} / L$ increased for later-type systems. Heidmann used data for 10 galaxies. The number of galaxies for which such data are available has been increased almost tenfold and the results are shown in Figure 14. Bottinelli et al. (1970) have recently determined an average $M_{H} / L$ value for 4 SO galaxies which fits nicely at the lower left of Figure 14 at a value a bit less than one half of the Sb value.

* See note added in proof, p. 34. 


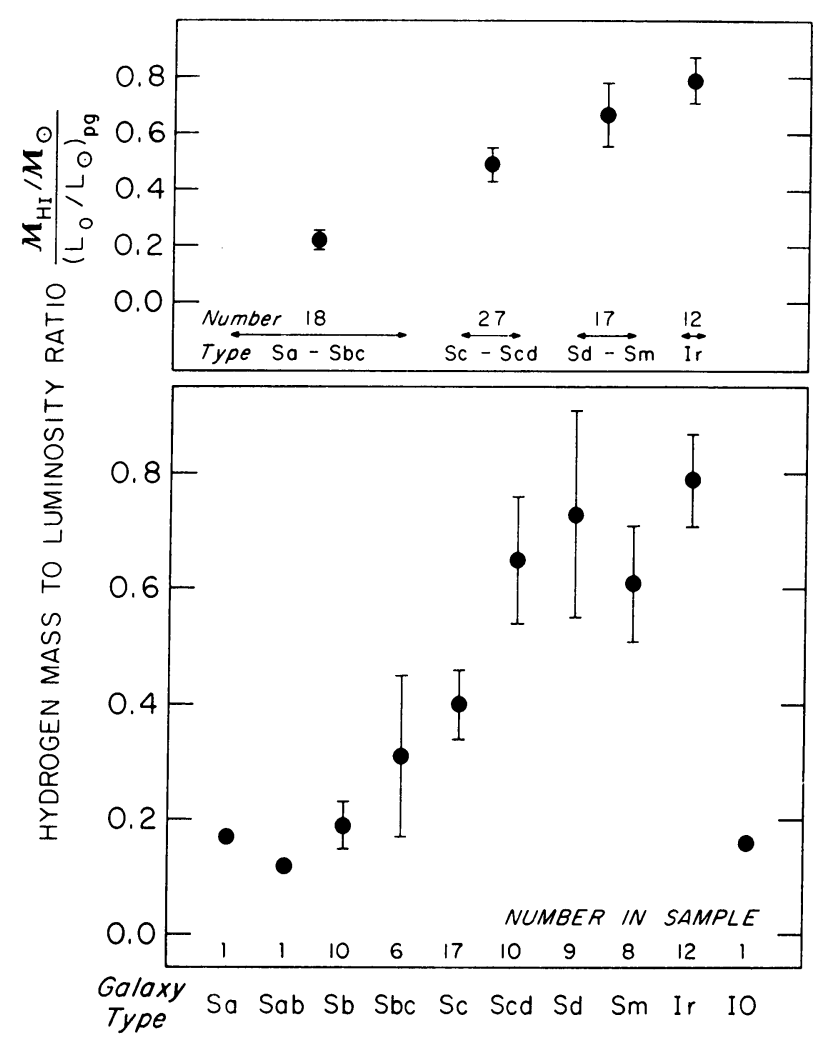

Fig. 14. The ratio: hydrogen mass to photographic luminosity for individual structural types (lower panel) and for broader type intervals (upper panel). The error bars represent the standard deviation from the mean. Both the hydrogen mass and luminosity are corrected for inclination effects.

The ratio of hydrogen mass-to-luminosity is a distance independent quantity so that this great uncertainty in extragalactic work is not present in these data. There is nevertheless a large range in $M_{H}$ for a given type and luminosity and conversely. This is shown in Figure 15, a plot of $M_{H}$ vs $L$. Some of this scatter reflects observational errors and the use of statistical corrections for a face-on orientation for the luminosity and hydrogen mass (Roberts, 1969). However, part of the range in $M_{H} / L$ values for a given type appears to be real and is correlated with the color of the galaxy as shown in Figure 16. Shown here is the color excess, $C_{0}-\left\langle C_{0}\right\rangle_{\text {type }}$, of a galaxy vs the normalized 'hydrogen excess' $\left[M_{H} / L-\left\langle M_{H} / L\right\rangle_{\text {type }}\right] /\left\langle M_{H} / L\right\rangle_{\text {type }}$. The 'error bars' in this figure show the full range in color at a particular abscissa value. This color dependence within a structural class appears to be another aspect of the general relation between $M_{H} / L$ with type and therefore with color.

Another correlation is found between the fractional hydrogen content of a galaxy and its structural type. This is shown in Figure 17. A detailed discussion of these integral properties of galaxies is given by Roberts (1969). 


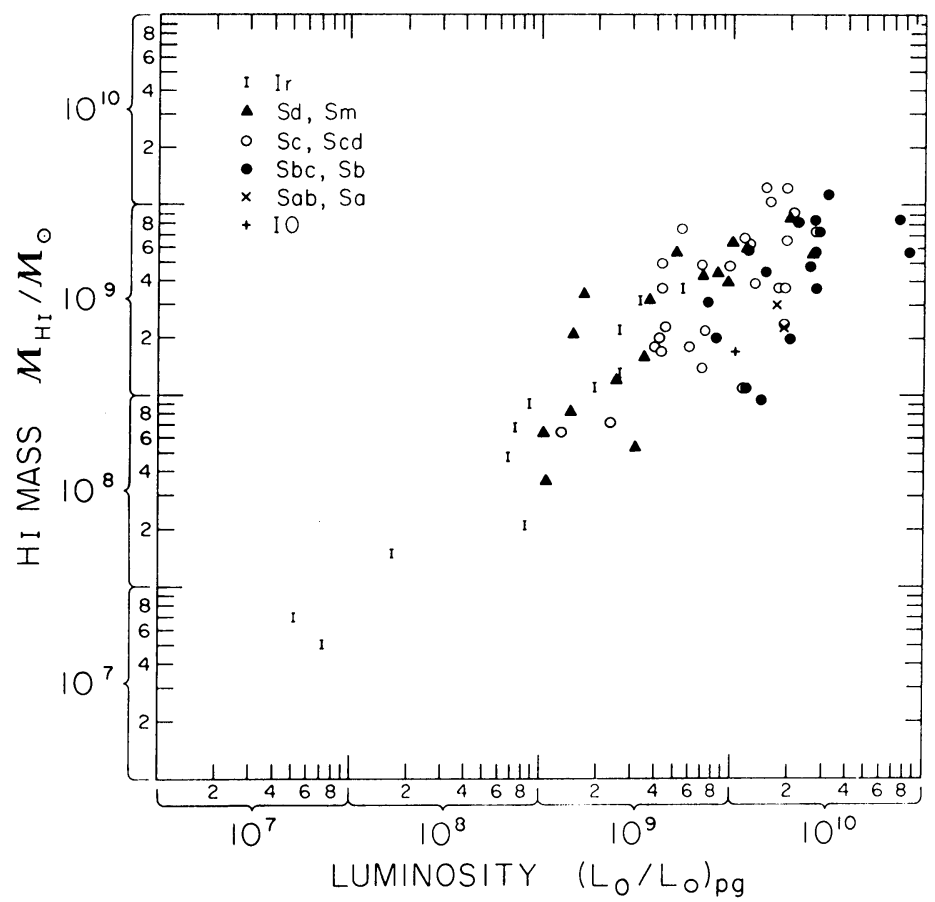

Fig. 15. The hydrogen mass-photographic luminosity relation for spiral and irregular-type galaxies. Both the hydrogen mass and luminosity are corrected for inclination effects.

The fractional hydrogen content of our Galaxy is about $5 \%$ (Kerr and Westerhout, 1965). The earlier value of 1 to $2 \%$ (Van de Hulst et al., 1957) has been significantly increased by a more detailed analysis.

These data on the hydrogen content of galaxies supply another aspect to the problem of the constancy of the helium-to-hydrogen abundance ratio discussed earlier. We find galaxies of the same structural type differing by a factor of $\sim 10$ in their hydrogen content, and therefore, presumably, in their helium content. We also find a similar range, going from one morphological class to another, in the percentage of the total mass of a galaxy that is in the form of neutral hydrogen and therefore in helium. We assume here that the helium is well mixed with the hydrogen throughout the total extent of the hydrogen. This assumption is necessary since the helium-tohydrogen ratio is determined only from $\mathrm{H}$ II regions which, as we saw earlier, are not uniformly distributed throughout the region of neutral hydrogen.

Of the two usual proposals invoked to account for the helium abundance: (i) primordial; or (ii) nucleosynthesis in stars, the former would appear the more attractive in terms of the different amounts of helium required in different galaxies. If we wish to invoke the nucleosynthesis explanation, we have the constraint of relating several parameters to the uncycled primordial plus cycled hydrogen (i.e., the present interstellar $\mathrm{HI}$ ). These parameters are the primordial mass function, the rate (and type) 


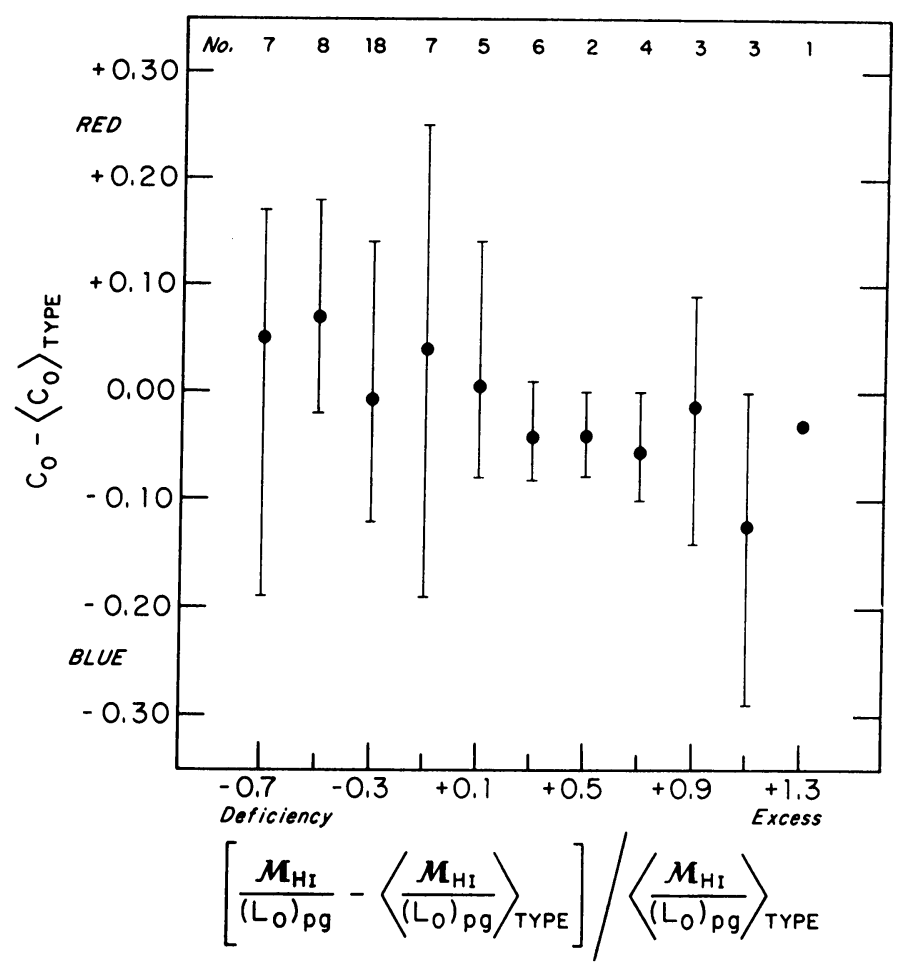

Fig. 16. The color excess of a galaxy vs the normalized hydrogen 'excess'. The excess in both parameters is with respect to the mean value for that type. The error bars represent the full range in color excess values. The hydrogen 'excess' values are averaged over intervals of 0.2 .

of later star formation and the amount and composition of the gas lost by evolving stars. In essence we require that the hydrogen and stellar content of a galaxy be related.

We have seen that the hydrogen content of a galaxy is well correlated with such galaxian properties as luminosity, total mass, color, and structural type. These are, in various ways, related to the stellar content of a galaxy and so the general requirement of a hydrogen-star relationship for the nucleosynthesis explanation is satisfied. However, the detailed relationships among the quantities listed above are not available without an adequate theory of star and galaxy formation and evolution. The approach may possibly be inverted and the relationships derived or at least indicated from the hydrogen and helium data.

\section{Neutral Hydrogen in Elliptical and Radio Galaxies}

Only one positive detection of $21-\mathrm{cm}$ line radiation from an elliptical galaxy, NGC 4472, has been reported. Robinson and Koehler (1965) derived a hydrogen mass of $3 \times 10^{8} M_{\odot}, M_{H} / L=0.007$ and $0.02 \%$ for the fractional mass content.

Hydrogen in absorption has been detected in the radio galaxy NGC 5128, Centaurus A (Roberts, 1970). This system is particularly favorable for such observations since 


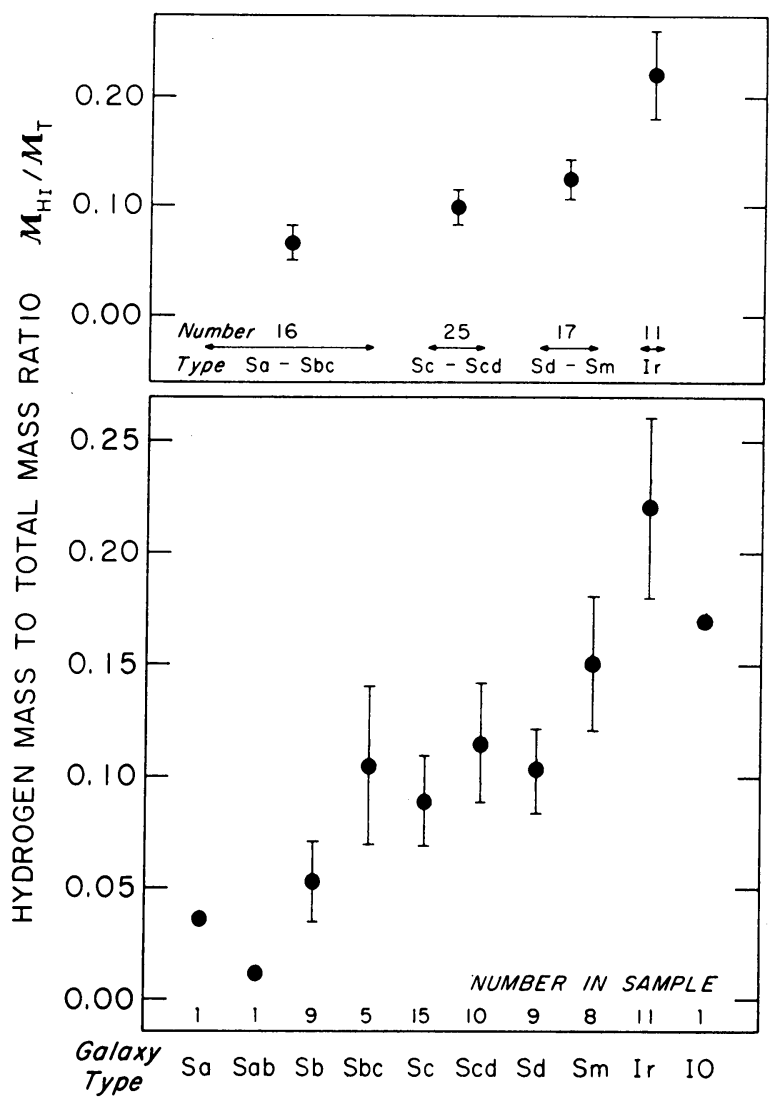

Fig. 17. The ratio:hydrogen mass to total mass for individual structural types (lower panel) and for broader type intervals (upper panel). The error bars represent the standard deviation from the mean.

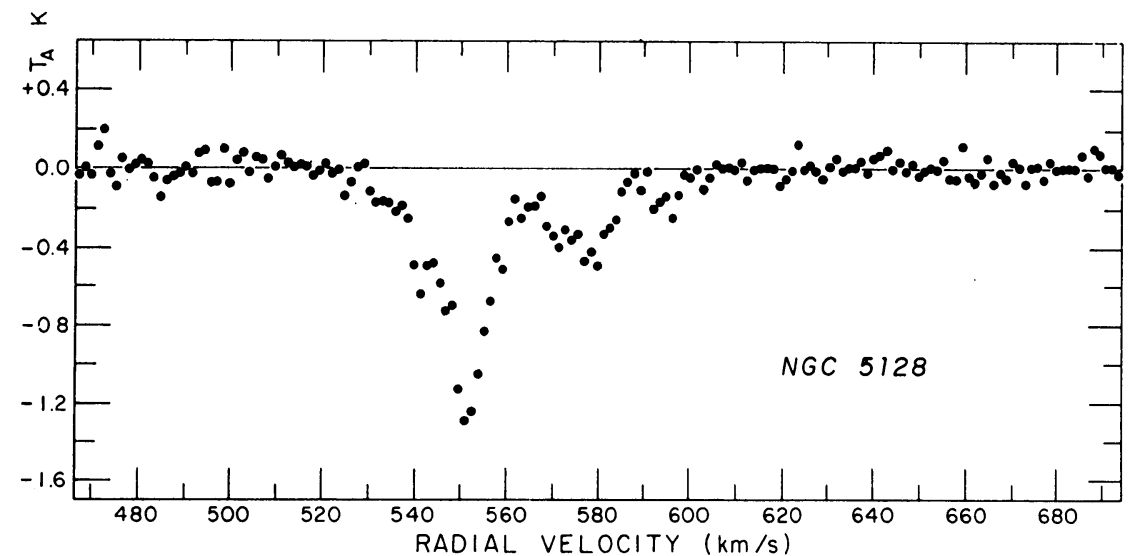

Fig. 18. Absorption velocity profile for Centaurus A, NGC 5128, obtained with the 140-foot telescope (Roberts, 1970). 
it is the only strong radio source in which much of the radio radiation comes from within the optical image of the galaxy. Further, the two central radio sources lie approximately on the rotation axis of the system so that the hydrogen seen projected on these sources is not subject to a rotational velocity spread.

The absorption feature, Figure 18, has at least 5 components. Its overall extent, at zero level, is $\sim 80 \mathrm{~km} \mathrm{~s}^{-1}$. The midpoint radial velocity is $563 \mathrm{~km} \mathrm{~s}^{-1}$. This may be compared with the optically derived systemic velocity of $540 \mathrm{~km} \mathrm{~s}^{-1}$. This latter value is based on the average of several emission line measurements. Absorption lines give a systemic velocity of $445 \mathrm{~km} \mathrm{~s}^{-1}$.

Little can be said about the optical depth of the absorbing features because their geometry is unknown. Clark (private communication) has pointed out that within the framework of several reasonable assumptions the absorption lifetime is $\sim 10^{3} \mathrm{yr}$, which is presumably unimportant with respect to the collision lifetime.

\section{Redshifts of Galaxies}

The previous discussion has dealt with observational material which yields information on the gaseous component of galaxies. The presence of such gas and its resultant spectral line radiation can also be used to study the motions within a galaxy as well as the systemic motion of a galaxy as a whole. A fundamental system of radial velocities, one essentially free of line blends, night sky emissions, and uncertain rest wavelengths, can be established with $21-\mathrm{cm}$ measurements. Such data can also be used to test the Doppler expression over a wavelength range of half a million by comparing optical and $21-\mathrm{cm}$ redshifts. We would expect a plot of these two quantities to have a slope of one and an intercept of zero. Such a comparison is shown in Figure 19. Because of blending with night sky $H$ and $K$ lines, those optical data taken in the blue part of the spectrum are found to be systematically too large by $\sim 100 \mathrm{~km} \mathrm{~s}^{-1}$ over the velocity range $\sim 1200 \mathrm{~km} \mathrm{~s}^{-1}$ to $\sim 2400 \mathrm{~km} \mathrm{~s}^{-1}$. These data are shown as an insert in Figure 19. Only red optical data are used for the full figure.

The mean of the two least-squares regressions for 130 velocity pairs over the range -400 to $+5200 \mathrm{~km} \mathrm{~s}^{-1}$ gives

$$
V(\mathrm{OPT})=-0.71+1.000 V(21-\mathrm{cm})
$$

We may conclude that the form of the Doppler expression is well confirmed over this velocity range.

A Hubble diagram similar to the classical $\left(m, c \Delta \lambda_{0} / \lambda_{0}\right)$ may be constructed from the $21-\mathrm{cm}$ data using $21-\mathrm{cm}$ flux density in place of apparent magnitude. Such a diagram with 117 entries is shown in Figure 20; the line shown is one with the expected slope of $\frac{1}{2}$. The Hubble constant can be evaluated from this diagram given a 'typical' galaxian hydrogen mass. Using the M81 group which has $7 \mathrm{HI}$ measurements, we obtain values of $\mathrm{H}$ of 83 to $109 \mathrm{~km} \mathrm{~s}^{-1} \mathrm{Mpc}^{-1}$ from solutions for different velocity intervals, i.e. all, $>500 \mathrm{~km} \mathrm{~s}^{-1}$, and $>1000 \mathrm{~km} \mathrm{~s}^{-1}$. Similar solutions for the 


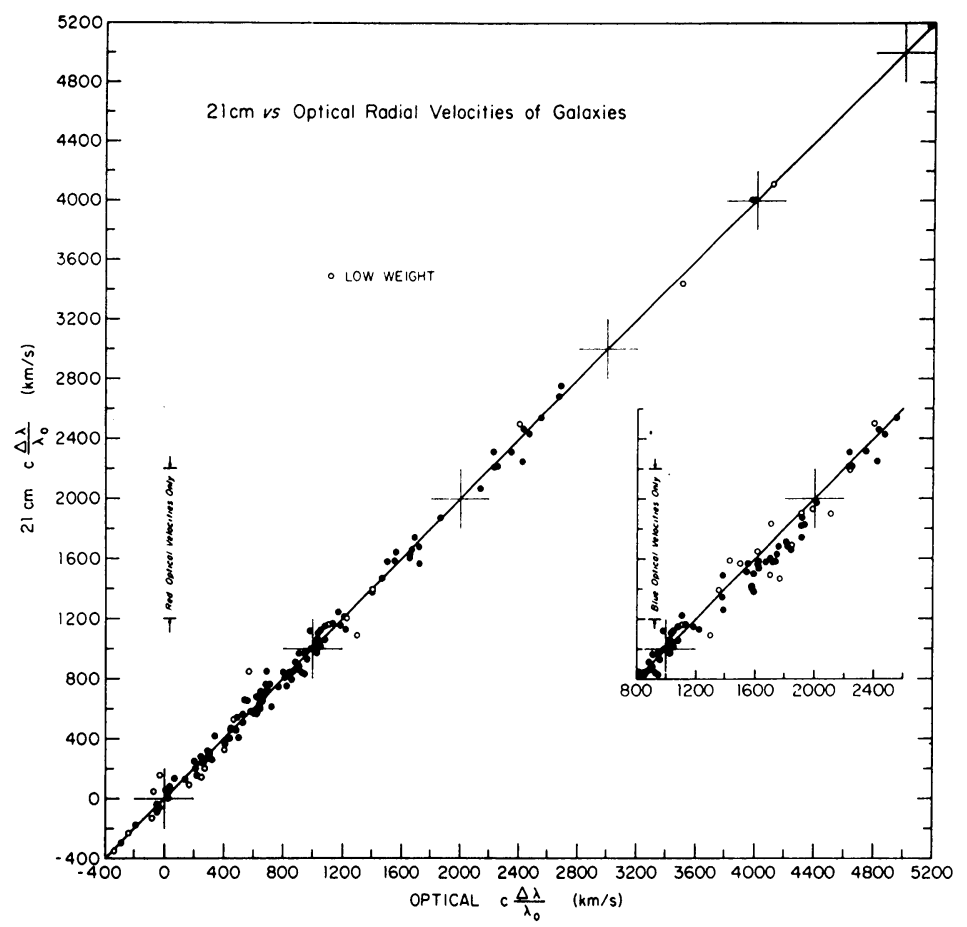

Fig. 19. Comparison of extragalactic redshifts measured at $21 \mathrm{~cm}$ and at optical wavelengths. The diagonal line has a slope of one and a zero intercept. Insert graph illustrates the systematic difference between $21-\mathrm{cm}$ and optical redshifts over the range $\sim 1200$ to $\sim 2400 \mathrm{~km} / \mathrm{s}^{-1}$ for optical values based on blue-sensitive spectra. The full graph has values in this velocity range based on red-sensitive spectra (primarily in the $\mathrm{H} \alpha$ region) and shows no systematic differences. The discrepancy with the blue optical data is most likely due to blends of night sky and galaxian $H$ and $K$ lines. The 21-cm data are from a variety of sources but are primarily based on Green Bank and Nançay observations.

Sculptor group, which has $5 \mathrm{HI}$ measurements, gives values of 78 to $103 \mathrm{~km} \mathrm{~s}^{-1} \mathrm{Mpc}^{-1}$. The solutions for $\mathrm{V}>500$ are to be favored since it has a relatively large number of galaxies, 85 , and is presumably free of non-isotropic effects in the velocity field (de Vaucouleurs, 1966). The Hubble constant values for these solutions are 100 (M81 group) and 94 (Sculptor group) $\mathrm{km} \mathrm{s}^{-1} \mathrm{Mpc}^{-1}$, giving an unweighted mean of $97 \mathrm{~km} \mathrm{~s}^{-1} \mathrm{Mpc}^{-1}$.

\section{Acknowledgements}

I wish to express my appreciation and thanks to colleagues with whom I have had valuable conversations regarding various topics covered in this review as well as those who have sent me material in advance of publication. Special acknowledgements are due to Drs. E. Churchwell, R. Hjellming, G. Huguenin, V. Rubin, and L. Searle. I am also indebted to Mr. A. H. Rots for his careful analysis and aid in preparing several of the figures and in carrying through the computations of the Hubble Constant from the $21-\mathrm{cm}$ data. 


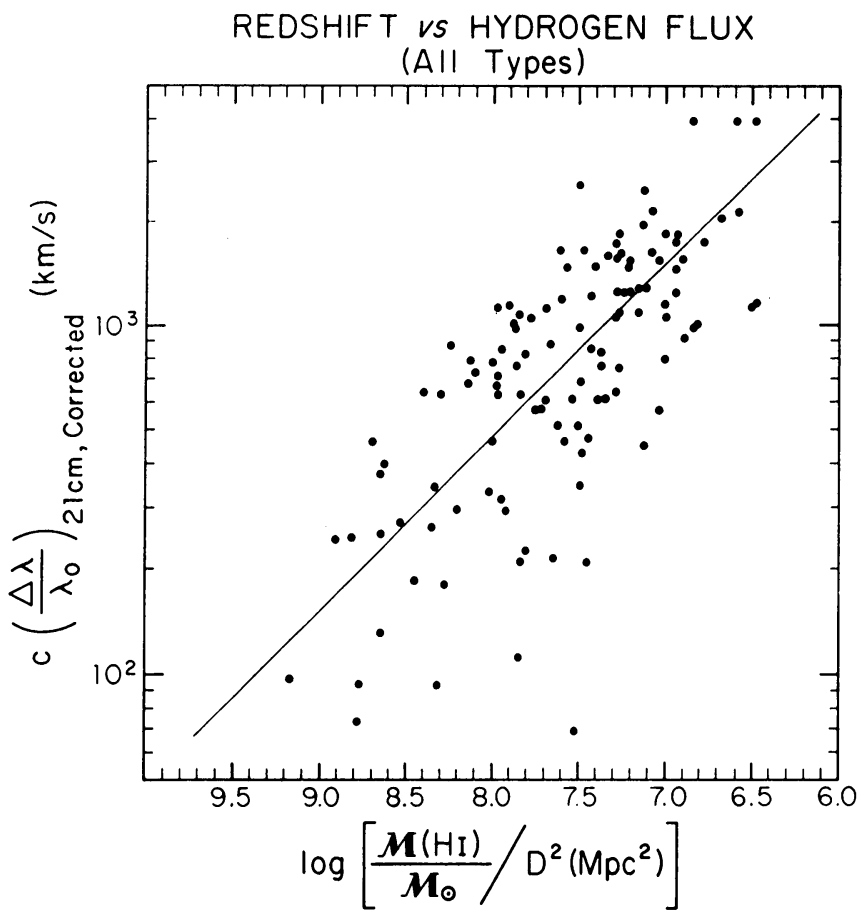

Fig. 20. The 'redshift-magnitude' relation based wholly on $21-\mathrm{cm}$ redshifts and magnitudes (i.e. hydrogen flux densities). The diagonal line has the expected slope of one half. To compare with its optical counterpart see the lower part of Figure 10 in Humason et al. (1956).

Note to page 27 added in proof. Dr F. Bertola informs me that he obtains a velocity of $-10 \mathrm{~km} \mathrm{~s}^{-1}$ for NGC 3077 . This value is based on three spectra $(80 \AA / \mathrm{mm})$ obtained with an image tube at the 200 -in. telescope. This new, low systemic velocity implies that the concentration of neutral hydrogen seen in the direction of NGC 3077 is most likely associated with NGC 3077 rather than being foreground galactic hydrogen. Thus the contours in Figures 11 and 12 should extend to and envelop NGC 3077.

\section{References}

Bottinelli, L., Chamaraux, P., Gouguenheim, L., and Lauqué, R.: 1970, Astron. Astrophys. 6, 453. Bridle, A. and Venugopal, V.: 1969, Nature 224, 545.

Burbidge, E. M. and Burbidge, G. R.: 1962, Astrophys. J. 135, 694.

Burbidge, E. M. and Burbidge, G. R.: 1965, Astrophys. J. 142, 634.

Burbidge, G. R., Gould, R. J., and Pottasch, S. R.: 1963, Astrophys. J. 138, 945.

Burke, B. F.: 1967, in H. van Woerden (ed.), 'Radio Astronomy and the Galactic System', IAU Symp. 31, 185.

Burke, B. F., Turner, K. C., and Tuve, M. A.: 1963, in Annual Report of the Director, Dept. of Terrestrial Magnetism, 1962-63, Carnegie Institute of Washington, Washington, D.C., p. 289.

Burns, R. and Roberts, M.: 1970, in preparation.

Carruthers, G. R.: 1970, Astrophys. J. Letters 161, L81.

Demoulin, M. T. H.: 1969, Astrophys. J. 157, 81.

Gordon, C.: 1970, Astron. J. 75, 914.

Gordon, K.: 1970, Astrophys. J. in press. 
Heidmann, J.: 1961, Bull. Astron. Inst. Neth. 15, 314.

Hindman, J. V., Kerr, F. J., and McGee, R. X.: 1963, Australian J. Phys. 16, 570.

Humason, M. L., Mayall, N. U., and Sandage, A. R.: 1956, Astron. J. 61, 97.

Johnson, H. M.: 1959, Publ. Astron. Soc. Pacific 71, 425.

Kerr, F. J. and Westerhout, G.: 1965, in G. P. Kuiper and B. M. Middlehurst (eds.), Galactic Structure, University of Chicago Press, Chicago, p. 167.

Lewis, B. M.: 1968, Proc. Astron. Soc. Australia 1, 104.

Lin, C. C.: 1970, Invited Discourse, 14th General Assembly IAU.

McGee, R. X.: 1964, Australian J. Phys. 17, 515.

McGee, R. X. and Milton, J. A.: 1966, Australian J. Phys. 19, 343.

Mathis, J. S.: 1965, Publ. Astron. Soc. Pacific 77, 90.

Mayall, N. U.: 1960, Ann. Astrophys. 23, 344.

Menon, T. K.: 1958, Astrophys. J. 127, 28.

Oort, J. H.: 1965, Trans. IAU, 12 A, 789, Figure 2.

Peimbert, M.: 1968, Astrophys. J. 154, 33.

Peimbert, M. and Costero, R.: 1969, Bol. Obs. Tonantzintla Tacubaya 5, 3.

Peimbert, M. and Spinrad, H.: 1970, Astrophys. J. 159, 809.

Reifenstein, E. C. III, Wilson, T. L., Burke, B. F., Mezger, P. G., and Altenhoff, W. J.: 1970, Astron. Astrophys. 4, 357.

Roberts, M. S.: 1968, Astrophys. J. 151, 117.

Roberts, M. S.: 1969, Astron. J. 74, 859.

Roberts, M. S.: 1970, Astrophys. J. Letters 161, L9.

Roberts, M. S. and Warren, J. L.: 1970, Astron. Astrophys. 6, 165.

Robinson, B. J. and Koehler, J. A.: 1965, Nature 208, 993.

Rubin, V. C., Ford, W. K., Jr., and D'Odorico, S.: 1970, Astrophys. J. 160, 801.

Schmidt, M.: 1962, in J. Sahade (ed.) 'Symposium on Stellar Evolution', Astronomical Observatory, La Plata, Argentina, p. 61.

Searle, L.: 1971, this volume, p. 66.

Shobbrook, R. R. and Robinson, B. J.: 1967, Australian J. Phys. 20, 131.

Turner, K. C.: 1970, in Annual Report of the Director, Department of Terrestrial Magnetism, 1968-69, Carnegie Institute of Washington, Washington, D.C., p. 366.

Van de Hulst, H. C., Raimond, E., and Van Woerden, H.: 1957, Bull. Astron. Inst. Neth. 14, 1.

Vaucouleurs, G. de: 1966, in G. Barbera (ed.) 'Galileo Symp. Cosmology', Florence, Italy, p. 37.

Volders, L. and Högbom, J. A.: 1961, Bull. Astron. Inst. Neth. 15, 307.

Weliachew, L.: 1969, Astron. Astrophys. 3, 402.

\section{Discussion}

Heidmann: I would like to add a few words to Morton Roberts' talk. Miss Bottinelli just completed her thesis on the large-scale distribution of neutral hydrogen in small galaxies. She obtained the effective $\mathrm{HI}$ diameter for $\mathbf{3 5}$ galaxies, from lenticulars to irregulars; this is the diameter containing half of the total neutral hydrogen. She found that the Hi diameter to optical Holmberg diameter ratio increases from one-half to one from $\mathrm{Sb}$ to Magellanic irregulars. Thus, the $\mathrm{HI}_{\mathrm{I}}$ is quite widely distributed and is more so for late types.

She found that one-third of these galaxies have an asymmetrical Hi distribution with a tendency for asymmetrical HI distributions to be associated with asymmetrical optical distributions. This may be related to the existence of HI companions pointed out by Roberts.

From the $\mathrm{HI}$ diameter, the true mean projected $\mathrm{HI}$ density can be deduced. There is practically no correlation of this true density with morphological type; its mean value is $1.3 \times 10^{-3} \mathrm{gm} \mathrm{cm}^{-2}$.

I would like to make a comment about Roberts' relation between color excess and $M_{H} / L$ excess. This may be related to the dependence of color index on morphological type and on intrinsic luminosity given in Gouguenheim's thesis (Astron. Astrophys. 3, 281, 1969). This may lead again to the fact that the main physical properties of galaxies are determined by type and luminosity as shown for optical diameters by myself (Astrophys. Letters 3,19, 1969) and for hydrogen masses and total masses by N. Heidmann (Astrophys. Letters 3, 153, 1969).

Arp: The results on the M81 system are exciting because they relate the M81 system to a class of galaxies where companions exist roughly on either side of a large galaxy. In many cases the 
companions are connected by luminous filaments to the main galaxy. Heretofore, no luminous connections have been observed between M81 and its two companions, but the radio observations just presented by Roberts indicate now that material in M81 extends in both directions towards the two peculiar companions, M82 and NGC 3077.

The importance of the association of two companions lies in the explanation for the cause of the connections. If it were hypothesized that the connection was the result of a perturbation caused by an encounter between the main galaxy and an orbiting companion, then it would be extremely unlikely that two such encounters would take place simultaneously on opposite sides of the main galaxy. Therefore, we must look elsewhere for an explanation of the fact that there is material linking the main galaxy and the companions.

Heidmann: In connection with comments on the M81 system, I would like to point out that Bottinelli found no relation between asymmetrical $\mathrm{HI}$ distributions and companion galaxies.

Mrs. Burbidge: I was surprised by the small velocity range given by the $\mathrm{HI}$ in NGC 5128; in the gas in the dust lane we found a considerably larger range due to rotation, from the $\mathrm{H} \alpha$ line.

Roberts: The 21-cm absorption arises from in front of the two prominent radio sources located within the optical image of NGC 5128. These sources lie near the axis of rotation of this galaxy which is approximately perpendicular to the dust lane. Thus, essentially no rotational component would be expected in the $21-\mathrm{cm}$ velocity profile.

Ekers: Perhaps there is a more widespread class of $\mathrm{HI}$ clouds without optical counterparts of which only 'companions' have been found while observing known galaxies.

Lewis: The velocity field of NGC 300 can be satisfactorily fitted by a single circulary symmetric rotation curve and optimized to fit the most appropriate major axis position angle. This is found to agree closely with the major axis defined by the integrated antenna temperature contours. Likewise, the results from the velocity field of NGC 5236 agree well with fitting an elliptic rotation curve to the data and treating as above. In this case the major axis of the outermost optical isophotes of Sersic and from the integrated antenna temperature contours all agree. Thus, it is not necessary to postulate any perturbations of the velocity field by companion galaxies. Indeed this is the conclusion I have also drawn from the M81-82 velocity field shown by Dr. Roberts. 